Referencia para citar este artículo: Muñoz-Muñoz, L. (2017). La autorregulación y su relación con el apego en la niñez. Revista Latinoamericana de Ciencias Sociales, Niñez y Juventud, 15 (2), pp. 807-821. DOI:10.11600/1692715x.1520201082016

\title{
La autorregulación y su relación con el apego en la niñez*
}

\author{
LORENA MUÑOZ-MUÑOZ ${ }^{* *}$
}

Profesora Universidad de Chile, Chile.

\section{Artículo recibido en agosto 1 de 2016; artículo aceptado en enero 7 de 2017 (Eds.)}

- Resumen (analítico): se presenta una revisión de la literatura empírica cuyo objetivo es describir y comparar los hallazgos de los estudios que relacionan apego con autorregulación en la infancia. Se demuestra que el diseño metodológico y la técnica de medición utilizada para evaluar tanto apego como autorregulación afectan los resultados obtenidos. El lapso temporal entre mediciones afecta los resultados en estudios longitudinales de autorregulación conductual, y la técnica de evaluación del apego afecta el resultado en investigaciones de autorregulación emocional. Los resultados coinciden en describir una relación entre patrones de apego y el uso de estrategias de autorregulación emocional, lo que no se replica para la relación entre apego y emocionalidad. Se alerta sobre cómo los diseños metodológicos afectan los resultados de las investigaciones y se propende por la utilización de conceptualizaciones más holística de autorregulación.

Palabras clave: autorregulación, apego, desarrollo infantil, metodología (Tesauro Isoc de Psicología).

\section{Self-regulation and its relationship to attachment in childhood}

- Abstract (analytical): the objective of the present article is to describe and compare empirical literature that investigates the relation between attachment and self-regulation in childhood. The analysis demonstrates that the methodological design and measurement technique used to evaluate both attachment and self-regulation have a significant affect on the results obtained. The time lapse between measurements affects results in longitudinal studies of behavioral self-regulation, and the method used to evaluate attachment affects the results in studies of emotional self-regulation. Despite the fact that the research analyzed is consistent in describing a relationship between attachment patterns and the use of certain emotional regulation strategies, there is no consistency in research on the relationship between attachment and emotionality. This literature review provides a warning on how methodological designs affect research results and suggests incorporating an holistic conception of self-regulation in future research.

Key words: self-regulation, attachment, child development, methodology (Isoc Psychology Thesaurus).

El presente artículo es de revisión y análisis de la literatura escrito entre el 1 Octubre del 2014 y el 30 Junio del año 2015. Es del área de las ciencias sociales, y subárea Psicología.

** Psicóloga. Magister en Psicología Clínica Infanto-juvenil de la Universidad de Chile. Actualmente cursa el Doctorado de Psicología en la Universidad deChile.Docente enelMagisterdePsicologíaEducacionaldelaUniversidad deChile. Orcid:0000-0002-2712-2492. ÍndiceH5:2. Correoelectrónico: lorenamunoz@ug.uchile.cl 


\section{A auto-regulação e sua relação com o apego na infância.}

- Resumo (analítico): o objetivo deste artigo é descrever e comparar a literatura empírica que investiga a relação entre apego e auto-regulação na infância. A análise demonstra que o desenho metodológico e a técnica de medição utilizada para avaliar ambos os conceitos afetam os resultados obtidos. O tempo entre as medições é importante para os estudos longitudinais da auto-regulação da conduta, além do mais o método usado para avaliar o apego é relevante para as investigações de auto-regulação das emoções. No entanto, os resultados coincidem em descrever uma relação entre os padrões de apego e o uso das estratégias de auto-regulação emocional, embora não acontece o mesmo para a relação entre o apego e a emotividade. A revisão alerta sobre como os desenhos metodológicos afetam os resultados da investigação e propõe incorporar no futuro, nas pesquisas sobre o tema, uma concepção holística da auto-regulação.

Palavras-chave: auto-regulação, apego, desenvolvimento da criança, desenhos metodológicos (Tesauro Isoc da Psicologia).

\section{-1. Introducción. -2. Método. -3. Resultados. -4. Conclusiones. -Lista de referencias.}

\section{Introducción}

Apego y autorregulación son dos conceptos ampliamente investigados en la etapa de la niñez. Es en la infancia cuando se establece el vínculo de apego (Bowlby, 1998) y también se adquieren los primeros mecanismos de autorregulación (Kopp, 1982). Tanto desde la teoría del apego como desde las teorías de autorregulación se conciben ambos constructos como íntimamente relacionados. La investigación empírica también ha relacionado apego con autorregulación en distintos contextos y desde distintas aristas conceptuales. La presente revisión busca describir y comparar los hallazgos de las investigaciones que abordan la relación entre autorregulación y apego en la niñez.

\section{Apego}

El apego alude a un sistema de conductas que se activa en situaciones de necesidad, y que busca mantener la proximidad y contacto con la persona con quien el sujeto posee un vínculo afectivo fuerte, que suele ser su cuidador principal (Bowlby, 1998). El apego se ha descrito como un sistema de regulación emocional que se desarrolla en la relación madre-hijo (Schore, 2000), planteándose que esta regulación funciona como prototipo para uturas regulaciones en la vida del individuo
(Sroufe, 2005). Más específicamente, el sistema de apego entre cuidador e infante participa en la regulación neurobiológica a través de la coregulación de los afectos positivos y negativos del recién nacido (Schore, 2001; Schore $\&$ Schore, 2007). Acciones como mecer al infante, tomarlo en brazos, o el contacto físico piel a piel se ha visto que ayuda a la regulación de los afectos del recién nacido (Calkin \& Leerkes, 2011). Desde la perspectiva de Fonagy y Target (2002), el contexto de las relaciones tempranas desarrolla en las personas un sistema de procesamiento de la información y control que facilita el desarrollo de los mecanismos de autorregulación.

Según la teoría del apego, el vínculo desarrollado entre cuidador y recién nacido da origen a conductas que pueden reflejar un patrón de apego seguro o un patrón de apego inseguro (Ainsworth, Blehar, Waters \& Wall, 1978). El patrón de apego seguro describe a un infante que percibe la disponibilidad del cuidador para ayudarlo en caso de necesitar protección o consuelo y que demuestra una apropiada conducta de exploración de su entorno (Weinfield, Sroufe, Egeland \& Carlson, 2008). En cambio, niños o niñas con un patrón de apego inseguro no tienen certeza respecto de la disponibilidad de sus cuidadores, o dudan respecto de la efectividad de la respuesta de éstos. Para adaptarse a este tipo de cuidadores los infantes pueden desarrollar conductas 
ansiosas o resistentes que llamen la atención de sus cuidadores, o pueden desarrollar conductas evitativas las que los protegen del rechazo de éstos (Weinfield et al., 2008). Con base en lo anterior, los patrones de apego se pueden clasificar en cuatro categorías: seguro, inseguro/ evitativo, inseguro/resistente (Ainsworth et al., 1978), y patrón de apego desorganizado (Main \& Solomon, 1990). Este último se refiere a un patrón de apego donde los infantes utilizan una serie de estrategias contradictorias entre sí.

\section{Autorregulación}

La autorregulación surge en un ambiente social en que la "hetero"-regulación (Sameroff, 2010), dada inicialmente por los cuidadores principales ocupa un rol central en el desarrollo de esta capacidad. Esto coincide con lo planteado por Vygotski (2009): "en el desarrollo cultural del niño, toda función aparece dos veces: primero a nivel social, y más tarde, a nivel individual; primero entre personas (interpsicológica), y después en el interior del propio niño (intrapsicológica)" (p. 94). El proceso de desarrollo de la autorregulación comienza desde el nacimiento, siguiendo distintas fases, hasta lograr un nivel de autorregulación más flexible que le permite al infante enfrentar contextos cambiantes, alrededor de los tres años de edad (Kopp, 1982).

La autorregulación es "una habilidad humana compleja y universal que estructura el comportamiento dirigido a metas e incrementa la probabilidad de satisfacer una variedad de necesidades individuales" (Trommsdorff, 2009, p. 687 [traducción propia]). La autorregulación es un término que abarca múltiples formas de funcionamiento que van desde las funciones fisiológicas a procesos mentales intencionales (Lerner, Lerner, Bowers, LewinBizan, Gestsdottir \& Brown-Urban, 2011). Esta diversidad posiblemente esté en la base de que estudios relacionados con la autorregulación utilicen una variedad de conceptos para aludir a ella, como: autocontrol, funciones ejecutivas, control inhibitorio, conciencia, posponer la gratificación, o estrategias de autorregulación emocional. Algunas estrategias de autorregulación emocional en niños y niñas pequeñas son por ejemplo calmarse a sí mismo, buscar ayuda, distraerse. Diversos autores han planteado que esta diversidad conceptual puede ser una de las problemáticas en esta área de estudio dada la falta de consenso respecto de su definición (Adrian, Zeman \& Veits, 2011; Bridges, Denham \& Ganiban, 2004; Cole, Martin \& Dennis, 2004).

Para abordarelestudio de la autorregulación, se suele distinguir entre autorregulación conductual y autorregulación emocional. La autorregulación emocional se define como los "procesos extrínsecos e intrínsecos responsables de monitorear, evaluar y modificar las reacciones emocionales, su intensidad y sus aspectos temporales, para la satisfacción de las metas personales" (Thompson, 1994, pp. 27-28 [traducción propia]). Por otra parte, la autorregulación conductual se define como la inhibición de las reacciones impulsivas del comportamiento para cumplir con las exigencias ambientales (Calkins, 2007).

\section{Medición oevaluación deautorregulación y apego}

Hay gran diversidad de técnicas y métodos de medición o evaluación para el apego y para la autorregulación. Así, en relación a la autorregulación, entre las técnicas utilizadas están los auto-reportes, los reportes contestados por profesores o cuidadores principales, las técnicas observacionales, los diseños experimentales y las evaluaciones biofisiológicas (Adrian et al., 2011).

Respecto al apego en la niñez, lo más común es evaluar los patrones de apego, ya sea con métodos categoriales o continuos. La Situación Extraña (Ainsworth \& Wittig, 1969) es el método categorial más valorado en evaluación de patrones de apego, y permite definir categorías de patrones de apego seguro e inseguro. Consta de observar la reacción del niño o niña y de su cuidador principal en una situación de entradas y salidas sucesivas del adulto de la sala donde está jugando el infante. El método más común para la evaluación 
continua del apego es el Attachment Q-Sort (Waters \& Deane, 1985), que en base a tarjetas, arroja el grado de conductas relacionadas con el patrón de apego seguro que tiene el infante.

\section{Relación entre apego y autorregulación}

Los estudios que vinculan apego con autorregulación, buscan describir o corroborar la relación entre un tipo de contexto afectivo co-regulatorio, es decir, un patrón de apego, con las conductas autorregulatorias que se observan en los infantes. Este tema de investigación ha tenido desarrollo en las últimas décadas, lo que ha permitido que existan artículos de revisión de la relación entre apego y autorregulación emocional (Brumariu, 2015; Calkins \& Leerkes, 2011). Entre los estudios hay coincidencia en que niños y niñas en edad escolar con patrón de apego seguro logran aplicar estrategias de autorregulación emocional efectivas, tanto con sus cuidadores principales como en contextos sociales donde la figura de apego no está presente (Brumariu, 2015; Calkins \& Leerkes, 2011). Sin embargo, la diversidad conceptual y metodológica dificulta extraer conclusiones certeras pese a la investigación existente.

La presente revisión tiene por objetivo dar cuenta de las discrepancias y coincidencias halladas entre los estudios que relacionan apego con autorregulación emocional y conductual. Para ello se analizarán los estudios según el concepto de autorregulación utilizado y según su metodología, de modo de vislumbrar si la consistencia en métodos o conceptos aporta a organizar los hallazgos de las investigaciones. Esto es relevante tanto para la construcción de la teoría que vincula estos constructos, como para dar una panorámica del estado actual de la investigación en este tema.

\section{Método}

Para dar cuenta del objetivo, se realizó una revisión sistemática de artículos empíricos publicados entre el año 2000 y el año 2014, cuyo tema principal fuese la vinculación entre apego y autorregulación conductual o emocional en niños y niñas de hasta 12 años de edad. Se realizó la búsqueda en las bases de datos Ebsco, Scielo, Scopus y Open Access. Para la búsqueda se usaron las palabras "apego" y "autorregulación", tanto en castellano como en inglés, como palabras claves y/o presentes en el título del artículo. Además se revisaron las referencias de los artículos en busca de otros artículos con la misma temática. Se excluyeron de la selección: a) aquellos artículos que no reportaban explícitamente la relación entre apego y autorregulación; b) aquellos artículos enfocados en la autorregulación del aprendizaje, ya que este es un ámbito específico y de carácter más bien cognitivo, no siendo del interés de la presente revisión; c) aquellos artículos que se enfocaban exclusivamente en aspectos regulatorios de la madre o el padre, pero no del infante; d) los artículos que utilizaban en su muestra personas con alguna característica no normativa, por ejemplo, prematurez. Se tomó esta decisión considerando que en esos casos pudiera haber otros factores influyendo en la relación entre apego y autorregulación; e) los artículos enfocados en niños y niñas con alguna patología o en el tratamiento clínico de afecciones.

Luego de la búsqueda se obtuvieron 25 estudios que cumplieron con los criterios mencionados (Tabla 1). 
Tabla 1. Artículos Seleccionados para la Revisión Ordenados según Fecha de Publicación y algunas Características de su Diseño.

\begin{tabular}{|c|c|c|c|c|c|}
\hline Artículos & País & $\begin{array}{l}\text { Nivel socio } \\
\text { económico }\end{array}$ & Diseño & $\begin{array}{l}\text { Edad de la } \\
\text { muestra }\end{array}$ & $\begin{array}{l}\text { Tipo de } \\
\text { Autorregulación }\end{array}$ \\
\hline $\begin{array}{l}\text { Contreras, J.; Kerns, K.; Weimer, } \\
\text { B.; Gentzler, A. \& Tomich, P. } \\
(2000)\end{array}$ & EE.UU. & Medio-alto & Transversal & 10 a 12 años & Emocional \\
\hline $\begin{array}{l}\text { Vondra, J.; Shaw, D.; Swearingen, } \\
\text { L.; Cohen, M. \& Owens, E. (2001) }\end{array}$ & EE.UU. & Bajo & Longitudinal & $\begin{array}{l}\text { entre } 12 \\
\text { meses y } 4 \\
\text { años }\end{array}$ & $\begin{array}{l}\text { Emocional y } \\
\text { Conductual }\end{array}$ \\
\hline $\begin{array}{l}\text { Braungart-Rieker, J.; Garwood, M.; } \\
\text { Powers, B. \& Wang, X. (2001) }\end{array}$ & $\mathrm{S} / \mathrm{I}$ & Medio & Longitudinal & $\begin{array}{c}4,12,13 \\
\text { meses }\end{array}$ & Emocional \\
\hline $\begin{array}{l}\text { Volling, B.; McElwain, N.; Notario } \\
\text { P. \& Herrera, C. (2002) }\end{array}$ & EE.UU. & Medio & Transversal & $\begin{array}{l}12 \text { y } 16 \\
\text { meses }\end{array}$ & Emocional \\
\hline $\begin{array}{l}\text { Gilliom, M.; Shaw, D.; Beck, J.; } \\
\text { Schonberg, M. \& Lukon J. (2002) }\end{array}$ & EE.UU. & Bajo & Longitudinal & $\begin{array}{l}1.5,3.5, \text { y } 6 \\
\text { años }\end{array}$ & $\begin{array}{l}\text { Emocional y } \\
\text { Conductual }\end{array}$ \\
\hline $\begin{array}{l}\text { Diener, M.; Mangelsdorf, S.; } \\
\text { McHale, J. \& Frosch, C. (2002) }\end{array}$ & EE.UU. & Medio & Transversal & 12 meses & Emocional \\
\hline Berlin, L. \& Cassidy, J. (2003) & EE.UU. & Medio -alto & Longitudinal & $\begin{array}{l}\text { entre } 15 \\
\text { meses y } 4 \\
\text { años }\end{array}$ & Emocional \\
\hline $\begin{array}{l}\text { Volling, B.; Blandon, A. \& Kolak, } \\
\text { A. (2006) }\end{array}$ & EE.UU. & Medio & Longitudinal & $\begin{array}{l}12,13,16 \\
\text { meses }\end{array}$ & Conductual \\
\hline $\begin{array}{l}\text { Smith, C. L.; Calkins, S. D. \& } \\
\text { Keane, S. P. (2006) }\end{array}$ & EE.UU. & Diverso & Transversal & 2 años & Emocional \\
\hline $\begin{array}{l}\text { Kerns, K.; Abraham, M.; } \\
\text { Schlegelmilch, A. \& Morgan, T. } \\
\text { (2007) }\end{array}$ & EE.UU. & $\mathrm{S} / \mathrm{I}^{*}$ & Transversal & 9 a 11 años & Emocional \\
\hline $\begin{array}{l}\text { Zimmermann P.; Mohr C. \& } \\
\text { Spangler G. (2009) }\end{array}$ & Alemania & Diverso & Transversal & 12 años & Emocional \\
\hline $\begin{array}{l}\text { Crugnola, R. C.; Tambelli, R.; } \\
\text { Spinelli, M.; Gazzotti, S.; Caprin, } \\
\text { C. \& Albizzati, A. (2011) }\end{array}$ & Italia & Diverso & Transversal & 13 meses & Emocional \\
\hline Colle, L. \& Del Giudice, M. (2011) & Italia & Medio & Transversal & 7 años & Emocional \\
\hline $\begin{array}{l}\text { Schwarz, B.; Stutz, M. \& } \\
\text { Ledermann, T. (2012) }\end{array}$ & Suiza & Medio -alto & Longitudinal & $\begin{array}{l}4^{\circ} \text { año } \\
\text { primaria }\end{array}$ & Emocional \\
\hline
\end{tabular}




\begin{tabular}{|c|c|c|c|c|c|}
\hline $\begin{array}{l}\text { Brenning, K.; Soenens, B.; Braet, C. } \\
\& \text { Bosmans, G. (2012) (se incluye } \\
\text { dado que la moda de la edad era de } \\
12 \text { años) }\end{array}$ & Bélgica & $\mathrm{S} / \mathrm{I}^{*}$ & Transversal & 12 a 14 años & Emocional \\
\hline $\begin{array}{l}\text { Brenning, K.; Soenens, B.; Braet, } \\
\text { C.; Bosmans, G. (2012) (se incluye } \\
\text { dado que el promedio de edad era } \\
\text { de } 12 \text { años) }\end{array}$ & Bélgica & $\mathrm{S} / \mathrm{I}^{*}$ & Transversal & 8 a 14 años & Emocional \\
\hline Leerkes, E. \& Wong, M. (2012) & EE.UU. & Diverso & Transversal & 16 meses & Emocional \\
\hline $\begin{array}{l}\text { Brumariu, L.; Kerns, K. \& Seibert, } \\
\text { A. (2012) }\end{array}$ & EE.UU. & Diverso & Transversal & 10 a 12 años & Emocional \\
\hline $\begin{array}{l}\text { Martins, E.; Soares, I.; Martins, C.; } \\
\text { Tereno, S. \& Osorio, A. (2012) }\end{array}$ & Portugal & Diverso & Longitudinal & $\begin{array}{l}10 \text { meses, } \\
12,16 \text { meses }\end{array}$ & Emocional \\
\hline $\begin{array}{l}\text { Heikamp, T.; Trommsdorff, G.; } \\
\text { Druey, M.; Hubner, R. \& von } \\
\text { Suchodoletz, A. (2013) }\end{array}$ & Alemania & Medio & Transversal & $\begin{array}{l}\text { entre } 4 \text { y } 6 \\
\text { años }\end{array}$ & Conductual \\
\hline $\begin{array}{l}\text { West, K.; Mathews, B. \& Kerns, K. } \\
\text { (2013) }\end{array}$ & EE.UU. & Medio & Longitudinal & $\begin{array}{l}15,24,36 \text { y } \\
54{\text { meses. } 1^{\circ}}^{\circ} \text { primaria }\end{array}$ & Conductual \\
\hline Kim, H. \& Page, T. (2013) & EE.UU. & Bajo & Transversal & $\begin{array}{l}3^{\circ} \text { a } 5^{\circ} \\
\text { primaria }\end{array}$ & Emocional \\
\hline $\begin{array}{l}\text { Roque, L.; Veríssimo, M.; } \\
\text { Fernandes, M. \& Rebelo, A. (2013) }\end{array}$ & Portugal & Diverso & Transversal & $\begin{array}{l}18 \text { a } 26 \\
\text { meses }\end{array}$ & Emocional \\
\hline $\begin{array}{l}\text { Drake, K.; Belsky, J. \& Fearon, R. } \\
\text { M. P. (2014) }\end{array}$ & EE.UU. & Medio & Longitudinal & 1 a 12 años & Conductual \\
\hline $\begin{array}{l}\text { Spangler, G. \& Zimmermann, P. } \\
\text { (2014) }\end{array}$ & Alemania & Diverso & Longitudinal & $\begin{array}{l}12 \text { meses, } 12 \\
\text { años }\end{array}$ & Emocional \\
\hline
\end{tabular}

*S/I: sin información.

Todos los estudios encontrados utilizaron una metodología cuantitativa, reportando sus resultados al menos en término de correlación significativa o no entre apego y autorregulación, además de utilizar métodos estadísticos más complejos.

\section{Resultados}

De forma preliminar resulta interesante constatar que la mayor parte de los estudios publicados se realiza en Estados Unidos (14 artículos) y en población europea (10 artículos). No se encontró ningún artículo que analice muestra latinoamericana. Así mismo, la mayor parte de las investigaciones estudian población de nivel socio-económico medio o medio alto (11 artículos), y solo tres estudios se enfocan específicamente en población de nivel socioeconómico bajo. Respecto al grupo etario que estudian, 16 investigaciones abordan desde los 0 a los 6 años, y nueve investigaciones incluyen niños y niñas entre los 6 y los 12 años. Respecto del diseño de investigación utilizado, 
la mayoría utiliza un diseño transversal, aunque nueve estudios plantean un diseño longitudinal. Finalmente, 21 estudios abordan la vinculación entre apego y autorregulación emocional, y solo seis abordan la relación entre apego y autorregulación conductual. El detalle se puede observar en la Tabla 1.

\section{Autorregulación conductual}

Los estudios que abordan la autorregulación conductual lo hacen desde diversas conceptualizaciones, como son control inhibitorio, problemas externalizantes, obediencia a reglas y adaptación a ámbitos sociales. Exceptuando el concepto de control inhibitorio, que suele evaluarse en contextos de laboratorio, hay cierta convergencia en evaluar la autorregulación conductual a partir de los reportes de personas significativas en los ámbitos naturales de los niños y niñas, como son las profesoras o las madres.

De los seis artículos que abordan el tema de la autorregulación conductual, solo una investigación utiliza un diseño transversal. Ésta encuentra que niños y niñas con apego seguro tienen mejor control inhibitorio que niños y niñas que no tienen apego seguro (Heikamp, Trommsdorff, Druey, Hubner \& von Suchodoletz, 2013).

Enlos estudios longitudinales, generalmente se busca evaluar si el tipo de apego que reporta el infante en su primera infancia se relaciona con su capacidad de autorregulación en años posteriores. A partir de la revisión, se encuentra que el patrón de apego en la infancia se relaciona con la capacidad de autorregulación conductual dependiendo de, a) la edad en que se evalúa el apego y, b) de la distancia temporal entre las mediciones de ambos constructos (Tabla 2).

Se reporta correlación significativa entre apego y autorregulación conductual cuando el tiempo transcurrido entre la medición de ambos constructos es de tres años o menos (Drake, Belsky \& Fearon, 2014; Vondra, Shaw, Swearingen, Cohen \& Owens, 2001; West, Mathews \& Kerns, 2013). En cambio, cuando la distancia entre mediciones es mayor a tres años, los estudios no encuentran correlación significativa entre patrón apego del infante y capacidad de autorregulación conductual, independientemente de cómo sea medida la autorregulación conductual (Drake et al., 2014; Gilliom, Shaw, Beck, Schonberg \& Lukon, 2002; Vondra et al. 2001; West et al., 2013).

Por otro lado, tampoco se encuentra correlación significativa entre apego y autorregulación conductual cuando el patrón de apego es evaluado previo a los 18 meses (Drake et al., 2014; Vondra et al., 2001; West et al., 2013). 
Tabla 2. Conceptualización y Distancia Temporal entre Mediciones en Resultados de Estudios que Abordan la Relación entre Apego y Autorregulación Conductual.

\begin{tabular}{|c|c|c|c|c|c|}
\hline Artículo & $\begin{array}{c}\text { Edad de } \\
\text { evaluación } \\
\text { del apego }\end{array}$ & $\begin{array}{c}\text { Edad de } \\
\text { evaluación de } \\
\text { autorregulación }\end{array}$ & $\begin{array}{l}\text { Correlación } \\
\text { significativa }\end{array}$ & $\begin{array}{l}\text { Distancia } \\
\text { temporal } \\
\text { entre } \\
\text { mediciones }\end{array}$ & $\begin{array}{c}\text { Concepto operacional } \\
\text { de Autorregulación } \\
\text { Conductual }\end{array}$ \\
\hline $\begin{array}{l}\text { Vondra et al. } \\
\text { (2001) }\end{array}$ & 24 meses & 3.5 años & Sí & 1.5 años & Problemas externalizantes \\
\hline $\begin{array}{l}\text { Drake et al. } \\
\text { (2014) }\end{array}$ & 36 meses & 6 años & Sí & 3 años & Autorregulación social \\
\hline $\begin{array}{l}\text { West et al. } \\
\text { (2013) }\end{array}$ & $\begin{array}{l}24 \text { y } 36 \\
\text { meses }\end{array}$ & 4.5 años & Sí & $\begin{array}{l}\text { Menos de } 3 \\
\text { años }\end{array}$ & $\begin{array}{l}\text { Control inhibitorio } \\
\text { Obediencia a reglas } \\
\text { escolares }\end{array}$ \\
\hline $\begin{array}{l}\text { Drake et al. } \\
\text { (2014) }\end{array}$ & $\begin{array}{l}15 \text { y } 36 \\
\text { meses }\end{array}$ & 4.5 años & Sí & $\begin{array}{l}\text { Menos de } 3 \\
\text { años }\end{array}$ & Control inhibitorio \\
\hline $\begin{array}{l}\text { Drake et al. } \\
(2014)\end{array}$ & $\begin{array}{l}15 \text { y } 36 \\
\text { meses }\end{array}$ & 6 años & No & $\begin{array}{l}\text { Más de } 3 \\
\text { años }\end{array}$ & Control inhibitorio \\
\hline $\begin{array}{l}\text { Drake et al. } \\
(2014)\end{array}$ & $\begin{array}{l}15 \text { y } 36 \\
\text { meses }\end{array}$ & 6 años & No & $\begin{array}{l}\text { Más de } 3 \\
\text { años }\end{array}$ & Persistencia en la tarea \\
\hline $\begin{array}{l}\text { Vondra et al. } \\
\text { (2001) }\end{array}$ & $\begin{array}{l}12 \text { o } 18 \\
\text { meses }\end{array}$ & 3.5 años & No & 2.5 años & Problemas externalizantes \\
\hline $\begin{array}{l}\text { Gilliom et al. } \\
\text { (2002) }\end{array}$ & 18 meses & 6 años & No & 4.5 años & Problemas externalizantes \\
\hline $\begin{array}{l}\text { West et al. } \\
\text { (2013) }\end{array}$ & 12 meses & 4.5 años & No & 3.5 años & Control inhibitorio \\
\hline $\begin{array}{l}\text { Heikamp et al. } \\
\text { (2013) }\end{array}$ & 4 - 6 años & $4-6$ años & Sí & $\begin{array}{l}0 \text { (estudio } \\
\text { transversal) }\end{array}$ & Control inhibitorio \\
\hline $\begin{array}{l}\text { Volling et al. } \\
\text { (2006) }\end{array}$ & 12 meses & 16 meses & $\begin{array}{l}\text { Depende del } \\
\text { apego con el } \\
\text { padre }\end{array}$ & 4 meses & Obediencia a los padres \\
\hline
\end{tabular}

Finalmente, un estudio realizado durante la primera infancia encuentra relación entre autorregulación conductual y apego con la madre solo cuando el niño o niña tenía apego inseguro con el padre (Volling, Blandon \& Kolak, 2006). Este resultado es interesante ya que plantea la necesidad de evaluar el apego con padre y madre para tener una mirada más completa de las situaciones que pueden estar incidiendo en la relación entre patrón de apego y autorregulación.

\section{Autorregulación emocional}

Como habíamos mencionado, en la conceptualización de la autorregulación emocional hay diferencias entre los investigadores. Entre los artículos revisados encontramos que algunos consideran dentro de las estrategias de autorregulación emocional a la expresión emocional (Smith, Calkins \& Keane, 2006; Volling, McElwain, Notario \& Herrera, 2002; Zimmerman, Mohr \& Spangler, 2009) o al reconocimiento emocional (Colle \& 
Del Giudice, 2011); mientras que otros separan estrategias de autorregulación emocional de emocionalidad (Contreras Kerns, Weimer, Gentzler \& Tomich, 2000). Además algunos utilizan el concepto de competencia emocional para aludir al reconocimiento de emociones y al conocimiento de estrategias de autorregulación emocional (Colle \& Del Giudice, 2011; Volling et al. 2002).

Para la presente revisión agruparemos los estudios en dos: estrategias de autorregulación emocional y emocionalidad. Siguiendo la tendencia de los estudios revisados, aquellos que aborden expresión emocional o reconocimiento emocional serán incluidos en las estrategias de autorregulación emocional.

\section{Emocionalidad: Afectividad negativa y positiva}

La emocionalidad se refiere a la frecuencia e intensidad con que los niños y niñas experimentan emociones con valencia positiva, como alegría o bienestar, o con valencia negativa, como rabia, temor o frustración (Contreras et al., 2000). Se suele operacionalizar como afectividad negativa o positiva. Los estudios que abordan la emocionalidad en su mayoría son de corte transversal y buscan corroborar si es que un patrón de apego inseguro está vinculado con mayor afectividad negativa. Para ello se utilizan técnicas observacionales o se recoge la información vía cuestionario. Los paradigmas observacionales son técnicas en que se expone al niño o niña a tareas que elicitan emociones, o simplemente se observa su juego libre mientras un evaluador puntúa la conducta comúnmente desde una pauta preestablecida. En estos estudios los hallazgos son disimiles dependiendo de la técnica utilizada y de si se evalúa afectividad positiva o negativa.

Los estudios que abordan la afectividad negativa y que utilizan técnicas observacionales encuentran que infantes con patrón de apego seguro presentan menor distres o emocionalidad negativa que infantes con patrón de apego inseguro (Diener, Mangelsdorf, McHale \& Frosch, 2002; Leerkes \& Wong, 2012; Smith et al., 2006; Volling et al., 2002). En cambio, las investigaciones que utilizan cuestionarios contestados por las madres para evaluar la afectividad negativa, no encuentran relación entre apego con la madre o el adulto y afectividad negativa (Contreras et al., 2000; Gilliom et al., 2002; Kim \& Page, 2013).

Solo dos estudios abordan la afectividad positiva, ambos desde técnicas observacionales. En menores de dos años no encuentran correlación significativa entre afectividad positiva y apego con la madre (Diener et al., 2002; Smith et al., 2006), en cambio sí se describe correlación significativa entre apego con el padre y afectividad positiva (Diener et al., 2002).

Finalmente, desde un estudio longitudinal predictivo, Braungart-Rieker, Garwood, Powers y Wang, (2001) encuentran que el afecto positivo evaluado a los 4 meses de edad, predice el patrón de apego con la madre y el padre a los 12 meses. Específicamente, infantes que puntúan alto en afectividad positiva con madres y padres a los 4 meses tienden a tener patrón de apego evitativo o seguro, en cambio quienes puntúan con baja afectividad positiva tienden a tener un patrón de apego resistente $o$ seguro, al año de edad.

\section{Estrategias de autorregulación emocional.}

La forma más recurrente para abordar el estudio de la autorregulación emocional es a través de las estrategias que se utilizan para ello. Mayoritariamente, tanto desde diseños transversales como longitudinales hay consenso en que niños y niñas utilizan estrategias de autorregulación emocional distintivas dependiendo de su patrón de apego.

Así, desde estudios longitudinales se describe que la conducta regulatoria evaluada antes del año de vida logra predecir el apego que tendrá el infante al año de edad (BraugartRieker et al., 2001; Martins, Soares, Martins, Tereno \& Osorio, 2012). Invirtiendo el orden de medición de los constructos, Gilliom et al. (2002) describen que una relación de apego seguro con la madre evaluado al año de edad logra predecir el uso de estrategias de autorregulación emocional consideradas adecuadas a los 3 años de vida. 
Sin embargo, estudios que utilizan diseños transversales con menores de dos años encuentran resultados disímiles dependiendo de la forma en que se evalúa el apego. Así, cuando se utiliza el método de la Situación Extraña, se encuentra relación entre patrón de apego y estrategias de autorregulación emocional (Crugnola et al., 2011; Diener et al., 2002; Leerkes \& Wong, 2012). Sin embargo, cuando se usa el instrumento Attachment Q-Sort para evaluar apego en menores de dos años, no se encuentra correlación entre apego y estrategias de autorregulación emocional (Smith et al., 2006), o se encuentra dependiendo del tipo de emoción que se quiere regular y de la disponibilidad de la madre (Roque, Veríssimo, Fernandes \& Rebelo, 2013).

En edad escolar, los artículos revisados tienden a coincidir en que existe relación entre apegoy estrategias de autorregulación emocional (Brenning, Soenens, Braet \& Bosmans, 2012; Colle \& Del Giudice, 2011; Contreras et al., 2000; Schwarz, Stutz \& Ledermann, 2012; Zimmermann, Mohr \& Spangler, 2009). Sin embargo, algunas investigaciones matizan estos hallazgos. Así, Brumariu, Kerns y Seibert (2012) reportan que niños y niñas con patrón de apego desorganizado usan menos estrategias de autorregulación emocional, pero no encuentran relación significativa en niños y niñas con otros patrones de apego y cantidad o tipo de estrategias de autorregulación. Spangler y Zimmermann (2014) encuentran correlación solo para regular la emoción de la rabia pero no para regular la emoción del temor, además, no encuentra correlación significativa entre patrón de apego y efectividad de la autorregulación emocional. Kerns, Abraham, Schlegelmilch y Morgan, (2007) encuentran correlación entre apego y estrategias de autorregulación emocional dependiendo de la forma de medir el apego. Ellos concluyen que probablemente las diferentes formas de evaluar apego apelan a diferentes facetas del constructo de apego.

En relación al reconocimiento emocional durante la etapa escolar, se describe que niños y niñas con patrón de apego seguro reportaron menor dificultad en identificar sus emociones (Brumariu et al., 2012), y que niños y niñas con patrón de apego desorganizado tienen levemente menor discriminación no verbal de emociones que los otros grupos de apego (Colle \& Del Giudice, 2011).

Finalmente, resulta interesante observar que la literatura de los últimos años, tiende a analizar la relación entre apego y estrategias utilizadas para distintos contextos emocionales (Brenning et al., 2012; Roque et al., 2013; Smith et al., 2006; Spangler \& Zimmermann, 2014). Este tipo de investigación, permite mayor especificidad en el conocimiento, aunque genera en ocasiones resultados confusos y difíciles de elaborar (Roque et al., 2013), o se tienden a diseños demasiado específicos (Brennings et al., 2012).

\section{Conclusiones}

La presente revisión sistemática tuvo como objetivo analizar la relación entre apego y autorregulación en niños y niñas descrita en los estudios empíricos de los últimos 15 años. Luego de esta revisión podemos concluir lo siguiente.

\section{a) Relación entre apego y autorregulación}

$\mathrm{La}$ vinculación entre apego y autorregulación establecida desde la teoría (Fonagy \& Target, 2002; Schore, 2000), a grandes rasgos se corrobora a nivel empírico, sin embargo los estudios dan cuenta de la multidimensionalidad de esta relación y de su volubilidad a lo largo de la niñez.

Por un lado, hay cierto consenso respecto de la vinculación entre patrón de apego seguro y el uso de mejores estrategias de autorregulación emocional. Esto se corroboró en algunos estudios longitudinales en preescolares y en estudios transversales con escolares, y coincide con revisiones previas de la relación entre apego y autorregulación emocional (Brumariu, 2015; Calkins \& Leerkes, 2011). Sin embargo, algunos de los estudios han puesto énfasis en que las estrategias de autorregulación emocional pudieran variar según el contexto y la emoción que se intenta regular. Este tipo de estudio está abriendo un nicho de mayor especificidad sobre el tema que pudiera aportar en la discusión respecto de la importancia del contexto social y 
emocional para la autorregulación y del alcance que tiene el apego para cada contexto.

Por otra parte, en menores de seis años, los estudios longitudinales dan cuenta que conductas autorregulatorias como son el control inhibitorio, la atención voluntaria, y otros componentes de las funciones ejecutivas, pueden ser moduladas por los procesos regulatorios aprendidos en el vínculo de apego. Sin embargo, esta relación se pierde cuando el tiempo entre la medición de ambos constructos es mayor a tres años, lo que puede implicar que otros factores comienzan a incidir una vez que los niños y niñas crecen o se insertan en nuevos contextos sociales.

Interesantes son los resultados respecto del tema de la emocionalidad. En edades escolares los estudios reportaron que no había relación entre apego y afectividad negativa, $\mathrm{y}$ en edades preescolares los resultados fueron diversos dependiendo de la metodología y de la muestra. Estos hallazgos pudieran aportar a la discusión respecto de si la emocionalidad es parte del proceso de autorregulación, o si por el contrario tiene mayor relación con aspectos del temperamento como sostienen algunos autores (Contreras et al., 2000; Rothbart, Ahadi, Hershey \& Fisher, 2001).

\section{b) Importanciadeldiseñometodológico utilizado.}

El diseño metodológico que se utiliza para estudiar la relación entre apego y autorregulación, sí afecta los resultados. Esto se observó tanto en las investigaciones que abordaron la autorregulación conductual como la autorregulación emocional.

En el caso de la autorregulación conductual, se constató que los diseños longitudinales obtienen correlación entre apego y autorregulación conductual dependiendo de la distancia temporal entre las mediciones. Este hallazgo puede dar cuenta del aspecto clave en el desarrollo infantil como es el interjuego entre continuidad y cambio (Sroufe, 2005). Particularmente, se ha descrito que la estabilidad en la categoría de apego, desde el año a edades preescolares es alrededor de un $45 \%$, siendo menor en población de nivel socioeconómico bajo (Vondra et al., 2001). Así mismo, evaluaciones de apego realizadas en edades posteriores suelen ser más predictivas que las de edades tempranas (Drake et al., 2014; Vondra et al., 2001). Esto concuerda con los hallazgos de esta revisión, en que se encontró relación entre apego y autorregulación conductual cuando la evaluación de apego se realizaba posterior a los 24 meses o en edades escolares.

$\mathrm{Al}$ analizar las investigaciones que abordan la relación entre apego y autorregulación emocional, fueron los instrumentos de evaluación utilizados los que afectaron los resultados encontrados. Así, en los estudios que vincularon apego con emocionalidad negativa se encontró relación solamente en los estudios que utilizaban técnicas observacionales, en que es un evaluador externo quien puntúa la afectividad negativa. No se encontró relación cuando se usó como técnica los cuestionarios o reportes contestados por la madre. Es decir, aparentemente, la visión que tiene la madre sobre la emocionalidad de su hijo o hija difiere de lo que reporta un observador externo. Si el reporte de la afectividad es del investigador, se encuentra que niños y niñas con apego seguro tienen menor afectividad negativa, en cambio, si es la madre quien reporta el grado de afectividad negativa de su hijo o hija, ese reporte no correlaciona con el patrón de apego del hijo o hija. Este hallazgo resulta relevante de considerar si queremos lograr investigaciones con validez ecológica, o que otorgue información aplicable en contextos naturales.

Así mismo, los estudios que vinculan apego con estrategias de autorregulación emocional en preescolares, encuentran correlación significativa solamente cuando la evaluación del apego se hace con el paradigma de la Situación Extraña y no cuando se usan otras técnicas de evaluación del apego. Esto recoge la discusión respecto de qué se mide cuando se evalúa apego con distintos instrumentos. Kerns et al. (2007) plantean que probablemente las diferentes formas de evaluar apego apelan a diferentes facetas del constructo de apego. En la misma línea, Martínez y Santelices (2005) discuten la diferencia entre evaluar una representación del apego (lo que se hace principalmente a través 
de entrevistas o cuestionarios) a evaluar lo que Bowlby llamó modelo interno operante, que se instaura como lo central de cada patrón de apego.

Por lo tanto, la elección del diseño metodológico, de la técnica de recolección de información, y de las edades en las que se desarrollará el estudio, debe ser pensado para reflejar coherentemente los propósitos de la investigación.

c) Investigaciones de laboratorio.

La mayoría de los estudios utilizó técnicas que se aplican en laboratorios (paradigmas observacionales para la evaluación del apego y de autorregulación y la situación extraña) además de cuestionarios. Este tipo de investigación es más controlable, sin embargo aleja los hallazgos de su aplicabilidad a contextos naturales. La diferencia en los resultados encontrados cuando responde la madre a cuando evalúa el investigador abre la pregunta respecto de qué se busca cuando se investiga, si lograr mediciones más objetivas o reflejar las vivencias subjetivas de nuestro sujeto de investigación.

En la misma línea, la tendencia en los estudios fue de indagar sobre aspectos parciales, particularmente de la autorregulación. Así lo que inicialmente se denominaba simplemente autorregulación (Kopp, 1982), luego pasó a ser diferenciado como autorregulación conductual y autorregulación emocional. La autorregulación conductual a su vez se estudia diferencialmente desde los conceptos de funciones ejecutivas, control inhibitorio y aspectos del temperamento (Zhou, Chen \& Main, 2011). La autorregulación emocional, se estudia distinguiendo las estrategias de autorregulación emocional de la respuesta emocional primaria (Koole, Van Dillen \& Sheppes, 2011), y últimamente ha aumentado el estudio de distintas estrategias de autorregulación según los distintos contextos emocionales. Esto permite lograr mayor especificidad y predictibilidad, sin embargo aleja la investigación del ser humano integral como sujeto de estudio. Las investigaciones futuras debieran intentar retomar una línea de investigación que considere la autorregulación como constructo complejo. Este tipo de investigación probablemente tendrá menor valor predictivo en sus resultados, sin embargo será más relevante para los contextos naturales y eventualmente sus resultados serán más aplicables.

Por otro lado, resulta relevante enfocar la autorregulación desde su perspectiva funcional, es decir de su efectividad para lograr las metas que el sujeto se ha propuesto (Bridges et al., 2004). Las estrategias de autorregulación pueden ser vista como inapropiadas por los adultos, pero pueden ser apropiadas para los niños cuyas metas pueden ser diferentes a las del adulto (Thompson, Lewis \& Calkins, 2008). Llama la atención que de los 25 estudios analizados, solo tres incorporaron autorreportes contestados por los propios niños y niñas para evaluar su autorregulación.

Por ende, futuras investigaciones debieran enfocarse en perspectivas más integrales de la autorregulación, con una metodología que permita incorporar los procesos de cambio propios del desarrollo infantil y que incorporen la visión de los niños y niñas considerando que la autorregulación depende de sus metas personales. De esta manera se podrá distinguir los alcances del apego para los procesos autorregulatorios en los contextos sociales naturales en que se desarrolla la infancia.

\section{Lista de referencias}

Las referencias marcadas con un asterisco indican estudios comprendidos en la revisión sistemática.

Adrian, M.; Zeman, J. \& Veits, G. (2011). Methodological implications of the affect revolution: A 35-year review of emotion regulation assessment in children. Journal of Experimental Child Psychology, 110, pp. 171-197. Doi:10.1016/j.jecp.2011.03.009.

Ainsworth, M. D. S.; Blehar, M. C.; Waters, E. \& Wall, S. (1978). Patterns of attachment: a psychological study of the Strange Situation. Hillsdale: Erlbaum.

Ainsworth, M. D. S. \& Wittig, B. A. (1969). Attachment and the exploratory behavior of one-year-olds in a strange situation. En B. M. Foss (ed.) Determinants of infant behavior IV, (pp. 111-136). London: Methuen. 
*Berlin, L. \& Cassidy, J. (2003). Mothers' self-reported control of their preschool children's emotional expressiveness: a longitudinal study of associations with infant-mother attachment and children's emotion regulation. Social Development, 12 (4), pp. 477-495.

Bowlby, J. (1998). El apego y la pérdida-1. El apego. Barcelona: Paidós.

*Braungart-Rieker, J.; Garwood, M.; Powers, B. \& Wang, X. (2001). Parental Sensitivity, Infant Affect, and Affect Regulation: Predictors of Later Attachment. Child Development, 72 (1), pp. 252-270.

*Brenning, K.; Soenens, B.; Braet, C. \& Bosmans, G. (2012). Attachment and depressive symptoms in middle childhood and early adolescence: testing the validity of the emotion regulation model of attachment. Personal Relationships, 19, pp. 445-464. Doi: 10.1111/j.14756811.2011.01372.x.

Bridges, L.; Denham, S. \& Ganiban, J. (2004). Definitional issues in emotion regulation research. Child Development, 75 (2), pp. 340-345.

Brumariu, L. E. (2015). Parent-child attachment and emotion regulation. New Directions for Child and Adolescent Development, (148), pp. 31-45. Doi:10.1002/cad.20098.

*Brumariu, L. E.; Kerns, K. \& Seibert, A. (2012). Mother-child attachment, emotion regulation, and anxiety symptoms in middle childhood. Personal Relationships, 19, pp. 569-585. Doi:10.1111/j.14756811.2011.01379.x.

Calkins, S. D. (2007). The emergence of selfregulation: Biological and behavioral control mechanisms supporting toddler competencies. In C. A. Brownell \& C. B. Kopp (eds.) Socioemotional development in the toddler years: transitions and transformations, (pp. 261-284). New York: Guilford Press.

Calkins, S. D. \& Leerkes, E. M. (2011). Early attachment processes and the development of emotional self-regulation. En K. D. Vohs \& R. F. Baumeister (eds.) Handbook of self-regulation: research, theory, and applications (2nd ed.), (pp. 355-373). New York: Guilford Press.
Cole, P.; Martin, S. \& Dennis, T. (2004). Emotion regulation as a scientific construct: methodological challenges and directions for child development research. Child Development, 75 (2), pp. 317-333.

*Colle, L. \& Del Giudice, M. (2011). Patterns of Attachment and Emotional Competence in Middle Childhood. Social Development, 20 (1), pp. 51-72. Doi: 10.1111/j.14679507.2010.00576.x.

*Contreras, J.; Kerns, K.; Weimer, B.; Gentzler, A. \& Tomich, P. (2000). Emotion Regulation as a Mediator of Associations Between Mother-Child Attachment and Peer Relationships in Middle Childhood. Journal of Family Psychology, 14 (1), pp. 111-124.

*Crugnola, R. C.; Tambelli, R.; Spinelli, M.; Gazzotti, S.; Caprin, C. \& Albizzati, A., (2011). Attachment patterns and emotion regulation strategies in the second year. Infant Behavior \& Development, 34,pp.136151. Doi:10.1016/j.infbeh.2010.11.002.

*Diener, M.; Mangelsdorf, S.; McHale, J. \& Frosch, C. (2002). Infants' Behavioral Strategies for Emotion Regulation with Fathers and Mothers: associations with Emotional Expressions and Attachment Quality. Infancy, 3 (2), pp. 153-174.

*Drake, K.; Belsky, J. \& Fearon, R. M. P. (2014). From early attachment to engagement with learning in school: the role of selfregulation and persistence. Developmental Psychology, 50 (5), pp. 1350-1361. Doi: 10.1037/a0032779.

Fonagy, P. \& Target, M. (2002). Early intervention and the development of selfregulation. Psychoanalytic Inquiry, 22, pp. 307-335.

*Gilliom, M.; Shaw, D.; Beck, J.; Schonberg, M. \& Lukon J. (2002). Anger Regulation in Disadvantaged Preschool Boys: Strategies, Antecedents, and the Development of Self-Control. Developmental Psychology, 38 (2), pp. 222-235. Doi: 10.1037//00121649.38.2.222.

*Heikamp, T.; Trommsdorff, G.; Druey, M.; Hubner, R. \& von Suchodoletz, A. (2013). Kindergarten children's attachment security, inhibitory control, 
and the internalization of rules of conduct. Frontiers in Psychology, 4, pp. 133-151. Doi: 10.3389/fpsyg.2013.00133.

*Kerns, K.; Abraham, M.; Schlegelmilch, A. \& Morgan, T.(2007). Mother-child attachment in later middle childhood: assessment approaches and associations with mood and emotion regulation. Attachment $y$ Human Development, 9 (1), pp. 33-53. Doi: 10.1080/14616730601151441.

*Kim, H. \& Page, T. (2013). Emotional Bonds with Parents, Emotion Regulation, and School-Related Behavior Problems Among Elementary School Truants. Journal of Child Family Studies 22, pp. 869-878. Doi:10.1007/s10826-012-9646-5.

Koole, S.; Van Dillen, L. \& Sheppes, G. (2011). Self-regulation of emotion. En K. D. Vohs \& R. F. Baumeister (eds.) Handbook of self-regulation: research, theory, and applications (2nd ed.), (pp. 22-40). New York: Guilford Press.

Kopp, C. (1982). Antecedents of selfregulation: a developmental perspective. Developmental Psychology, 18 (2), pp. 199-214.

*Leerkes, E. \& Wong, M. (2012). Infant Distress and Regulatory Behaviors Vary as a Function of Attachment Security Regardless of Emotion Context and Maternal Involvement. Infancy, 17 (5), pp. 455-478. Doi: 10.1111/j.15327078.2011.00099.x.

Lerner, R.; Lerner, J.; Bowers, E.; LewinBizan, S.; Gestsdottir, S. \& Brown-Urban, J. (2011). Self-regulation processes and thriving in childhood and adolescence: a view of the issues. New Directions for Child and Adolescent Development, (133), pp. 1-9.

Main, M. \& Solomon, J. (1990). Procedures for identifying disorganized/disoriented infants during the Ainsworth Strange Situation. In M. Greenberg, D. Cicchetti \& M. Cummings (eds.) Attachment in the preschool years, (pp. 121-160). Chicago: University of Chicago Press.

Martínez, C. \& Santelices, M. (2005). Evaluación del apego en el adulto: una revisión. Psykhe, 14 (1), pp. 181-191. Doi: 10.4067/S0718-222820050001000280.

*Martins, E.; Soares, I.; Martins, C.; Tereno, S. \& Osorio, A. (2012). Can we identify emotion overregulation in infancy? Infant and Child Development, 21, pp. 579-595. Doi: 10.1002/icd.1760.

*Roque, L.; Veríssimo, M.; Fernandes, M. \& Rebelo, A. (2013). Emotion regulation and attachment: relationships with children's secure base, during different situational and social contexts in naturalistic settings. Infant Behavior and Development, 36, pp. 298306. Doi: 10.1016/j.infbeh.2013.03.003.

Rothbart, M. K.; Ahadi, S. A.; Hershey, K. L. \& Fisher, P. (2001). Investigations of temperament at three to seven years: The Children's Behavior Questionnaire. Child Development, 72, pp. 1394-1408.

Sameroff, A. J. (2010). A unified theory of development: a dialectic integration of nature and nurture. Child Development, 81 (1), pp. 6-22. http://doi.org/10.1111/j.14678624.2009.01378.x.

Schore, A. (2000). Attachment and the regulation of the right brain. Attachment \& Human Development, 2 (1), pp. 23-47. http://doi.org/10.1080/146167300361309.

Schore, A. (2001). Effects of a secure attachment relationship on right brain development, affect regulation, and infant mental health. Infant Mental Health Journal, 22 (1-2), pp. 7-66.

Schore, J. \& Schore, A. (2007). Modern Attachment Theory: The Central Role of Affect Regulation in Development and Treatment. Clinical Social Work Journal, 36 (1), pp. 9-20. Doi:10.1007/s10615-0070111-7.

*Schwarz, B.; Stutz, M. \& Ledermann T. (2012). Perceived interparental conflict and early adolescents' friendships: the role of attachment security and emotion regulation. Journal of Youth and Adolescence, 41, pp. 1240-1252. Doi: 10.1007/s10964-0129769-4.

*Smith, C. L.; Calkins, S. D. \& Keane, S. P. (2006). The relation of maternal behavior and attachment security to toddlers' emotions and emotion regulation. Research 
in Human Development, 3 (1), pp. 21-31. Doi:10.1207/s15427617rhd03013.

*Spangler, G. \& Zimmermann, P. (2014). Emotional and adrenocortical regulation in early adolescence: prediction by attachment security and disorganization in infancy. International Journal of Behavioral Development, 38, pp. 1-13. Doi: $10.1177 / 0165025414520808$.

Sroufe, L. (2005). Attachment and development: A prospective, longitudinal study from birth to adulthood. Attachment y Human Development, 7 (4), pp. 349-367. Doi:10.1080/14616730500365928.

Thompson, R. A. (1994). Emotion regulation: a theme in search of definition. Monographs of the Society for Research in Child Development, 59 (2-3), pp. 25-52.

Thompson, R. A., Lewis, M. D. \& Calkins, S. D. (2008). Reassessing emotion regulation. Child Development Perspectives, 2 (3), pp. 124-131.

Trommsdorff, G. (2009). Culture and Development of Self-Regulation. Social and Personality Psychology Compass, 3 (5), pp. 687-701. http://doi.org/10.1111/ j.1751-9004.2009.00209.x.

*Volling, B.; Blandon, A. \& Kolak, A. (2006). Marriage, Parenting, and the Emergence of Early Self-Regulation in the Family System. Journal of Child and Family Studies, 15 (4), pp. 493-506. Doi: 10.1007/ s10826-006-9027-z.

*Volling, B.; McElwain, N.; Notario P. \& Herrera, C. (2002). Parents' emotional availability and infant emotional competence: predictors of parent-infant attachment and emerging self-regulation. Journal of Family Psychology, 16 (4), pp. 447-465.

*Vondra, J.; Shaw, D.; Swearingen, L.; Cohen, M. \& Owens, E. (2001). Attachment stability and emotional and behavioral regulation from infancy to preschool age. Development and Psychopathology, 13, pp. 13-33.

Vygotski, L. (2009). El desarrollo de los procesos psicológicos superiores. Buenos Aires: Crítica.
Waters, E. \& Deane, K. (1985). Defining and assessing individual differences in attachment relationships: Q-methodology and the organization of behavior in infancy and early childhood. In I. Bretherton \& E. Waters (eds.) Growing points of attachment theory and research. Monographs of the Society for Research in Child Development, 50 (209), pp. 41-65.

Weinfield, N.; Sroufe, A.; Egeland, B. \& Carlson, E. (2008). Individual differences in infant-caregiver attachment: conceptual and empirical aspects of security. En J. Cassidy \& P. R. Shaver (eds.) Handbook of attachment (2nd ed.), (pp. 78-101). New York: Guilford.

*West, K., Mathews, B. \& Kerns K. (2013). Mother-child attachment and cognitive performance in middle childhood: an examination of mediating mechanisms. Early Childhood Research Quarterly, 28, pp. 259-270. Doi:10.1016/j. ecresq.2012.07.005.

Zhou, Q.; Chen, S. H. \& Main, A. (2011). Commonalities and differences in the research on children's effortful control and executive function: a call for an integrated model of self-regulation. Child Development Perspectives, 6 (2), pp. 112-121. Doi: 10.1111/j.17508606.2011.00176.x.

*Zimmermann, P.; Mohr, C. \& Spangler, G. (2009). Genetic and attachment influences on adolescents' regulation of autonomy and aggressiveness. Journal of Child Psychology and Psychiatry, 50 (11), pp. 1339-1347. 